Session

\title{
Teaching Biology from an Engineering Perspective: Integrating Biology into Undergraduate Engineering Education
}

\author{
Kjell Nelson', Robin Adams ${ }^{1}$, Mary Lidstrom², Jeff Bonadio ${ }^{3}$, Dave Stahl ${ }^{4}$, \\ Cynthia Atman ${ }^{1}$ \\ ${ }^{1}$ Center for Engineering Learning and Teaching, ${ }^{2}$ Department of Chemical \\ Engineering, ${ }^{3}$ Department of Bioengineering, ${ }^{4}$ Department of Civil and \\ Environmental Engineering, University of Washington, Seattle, Washington
}

\begin{abstract}
A new course has been developed at the University of Washington that is designed to teach fundamental biological concepts specifically to engineering students who have little or no college level biology background. This course, entitled "Biological Frameworks for Engineers" instructs biology concepts from an engineering perspective and emphasizes the functional aspects of biological systems. It is divided into a series of three to four week modules, each establishing a real-world problem as a context for active, problem-based learning. A combination of instructional methods are used for each module such as lectures, in-class studentcentered exercises, discussions, and labs. A matrix of assessment methods are integrated into the curriculum to triangulate on student learning outcomes and to provide feedback to the instructional team as part of an iterative course development cycle. This paper describes the content, implementation, and assessment strategies of the first two course offerings, and describes the key initial findings.
\end{abstract}

\section{Introduction}

Scientific and technological advances during the $20^{\text {th }}$ century have shifted the emphasis of the US economy from manufacturing to information, communication, and high technology. Continuing US economic and technological leadership will rely in part on engineers who can recognize, understand, and integrate new developments in information technology, nanotechnology, and the life sciences. Engineering education programs must be responsive to these trends and adapt existing curricula to train new generations of engineers who are able to fulfill the changing needs of our society.

A significant growth area for future engineering programs is the interface between engineering and biology. This is occurring because of the revolution in the biological sciences brought about by rapid advances in molecular biology and the availability of genome sequences, the move toward ever smaller and more complex systems, and the necessity of responding to the pressures 
civilization is placing on vital global ecosystems. Several different areas are emerging at the interface between engineering and biology, including 1) the engineering of biological materials and processes, 2) the development of technology for biological measurements, and3) the application of biological paradigms to engineered systems. Although diverse, these fields are all linked by the pressing need for engineers to understand fundamental concepts in biology. The concurrence of these forces has propelled us towards biology as a new frontier for engineering education.

To meet this need, the authors, faculty in the UW College of Engineering who are experts in biology together with educational researchers from the UW Center for Engineering Learning and Teaching are developing a series of new courses and smaller curriculum modules designed to integrate biology into undergraduate engineering education at the UW. Our ultimate aim is to integrate biology into the engineering curriculum at three levels. This includes: 1) developing a set of instructional modules that use biological examples to illustrate engineering principles for incorporation into existing freshman or sophomore level engineering science courses 2) a junior level course in fundamental biological principles, and 3) advanced courses in engineering biological systems for seniors and graduate students. For our first effort in this program, we developed a course to teach fundamental biological concepts that are sufficiently rigorous and relevant to third and fourth year engineering students.

This paper describes the broad objectives, course content, implementation strategies, assessment methods, and initial key findings from this course, entitled Biological Frameworks for Engineers. We begin by describing the initial pilot course, discuss our findings from the pilot offering, and end with revisions implemented for the first full course offering given Autumn 2001.

\section{Biological Frameworks for Engineers}

\section{Course description}

"Biological Frameworks for Engineers", first piloted in spring quarter 2001, is currently offered each autumn quarter. The target audience is junior or senior Chemical Engineering and Civil and Environmental Engineering students (although all disciplines are encouraged to enroll) who have completed little or no prior college biology coursework. The prerequisites are calculus through differential equations, thermodynamics, inorganic chemistry, and elementary computer programming. Course goals include: 1) instruct a selected set of fundamental biological facts and concepts, 2) help students develop skill in applying these concepts to novel situations, 3) stimulate students' interest in biology and in further pursuing biology knowledge relevant to their major, and 4) demonstrate the importance and relevance of biology skills and knowledge for engineers practicing in the $21^{\text {st }}$ century. Biological concepts are taught in a language designed to be accessible to students with diverse engineering backgrounds. The curriculum is divided into discreet yet interrelated three to four week modules, each of which uses a specific real-world problem as a framework to explore and apply the biology concepts that are being introduced. The modules blend lectures, in-class exercises, and labs to deliver content knowledge, help students assimilate new knowledge in a student-centered, social setting, and ground the techniques they've learned in class in a hands-on setting (respectively.) Assessment 
instruments are integrated into the curriculum as much as possible and are designed to evaluate: 1) students' understanding of the concepts presented, 2) their ability to apply new knowledge and skills to novel situations, and 3) their attitudes about learning and applying biology concepts as engineers. Course development is iterative; the content and assessment strategies are modified in response to the results of the previous quarter's assessment results. Following a cycle of assessment, review, and refinement, "free standing" modules will be developed that could be readily exported to other programs, departments, or universities.

\section{Instructional Approach}

Whereas "traditional" biology courses emphasize memorization of facts, our course is designed to appeal to engineering students who are accustomed to learning how to use and apply tools and concepts in a "hands-on" setting. To teach biology to such students, our course was designed along the following guiding principles: 1) teach biology from an engineering perspective to bridge new biological concepts to students' engineering skills and existing cognitive frameworks, 2) establish a relevant, timely, interesting real-world framework for learning, and applying new facts and concepts, 3) provide biological facts and concepts as tools for understanding and solving the "puzzles" given within each module's framework, 4) monitor students' progress and assist the assimilation and accommodation of biological principles when their engineering frameworks are inadequate, and 5) motivate and engage students by using active, student-centered exercises, emphasizing the functions and integration inherent in biological systems, and relating the content as much as possible to engineering systems.

In short, our course is designed to help students acquire fundamental biological principles and assimilate them into their existing conceptual frameworks by drawing parallels between biology and engineering. By doing so within a problem-based setting, the students will both learn new facts and concepts as well as gain experience applying these concepts to novel settings. Along the way, we hope to demonstrate how their existing skills make them uniquely qualified to solve emerging problems at the biology / engineering interface and motivate them to pursue such problems in their professional careers.

\section{Course Themes and Content}

The following course themes were articulated to meet our goal of teaching biology from an engineering perspective. These themes are inherent in the context of each modules' contextual framework:

- $\quad$ living systems are the manifestation of Nature's engineering

$\circ$ the behavior of biological systems is determined by the same physical and chemical properties that apply to human-engineered systems.

- $\quad$ evolution is Nature's design tool

o in contrast to human-engineered systems, natural systems sample their "design space" through small changes in existing systems. Success is determined by how well these changes fit into the physical, chemical, and mechanical nature of the universe.

- $\quad$ living systems are founded on thermodynamic principles 
- organisms are far from entropic or chemical equilibrium and therefore must utilize energy to produce structure and maintain function. Death is, in a sense, equivalent to returning to thermodynamic equilibrium.

- living systems are integrated at the molecular, cellular, organismal, and ecosystem levels

- whereas human-engineered systems can function discreetly, natural systems are interconnected to each other at all levels within their environment and are sensitive to changes in seemingly unrelated areas.

- structure and function are related at all scales and levels of biological systems

○ the structure and function of biological systems can be analyzed in the context of physical principles familiar to engineers. Studying the physical architecture of a biological system can lead to insights into its function based on a few key biological principles which can be used to characterize living systems.

These abstract concepts were used guide the instruction of the concrete physical processes that were chosen as fundamental biology knowledge. The concrete processes chosen include:

- biological information storage and processing (the central dogma)

- genome organization and genomics

- biological system integration at the cellular, tissue, organism, and ecosystem levels

- disease and etiology

- evolution / diversification

- biological energy conversion and utilization (bioenergetics)

- biological signaling and communication

- legal, ethical issues related to biotechnology

Having outlined the course strategies and content, the instructors then chose the "problems"; settings that would fit within their expertise and integrate the course themes and fundamental concepts as outlined above.

\section{Course Content and Module Organization}

Course modules were developed to 1) provide a contextual framework from within which to illustrate and establish the relevance of the core biology knowledge and to understand and solve the problems posed, and 2) helping students develop skill as engineers operating at the biology / technology interface and applying their knowledge to novel situations. The modules, therefore, are fairly complex, open-ended, and last several weeks, which allows the students enough time to learn and apply course concepts, as well as minimizing "instructional overhead."

Module 1: The functions of life and the design space of biological systems. This first in-class exercise was designed to elicit students' conceptions of biological systems. The question was posed: "What are the functions of living systems?" Students were encouraged to share their ideas with the class and generate a list of responses. This list was grouped into categories by the instructor who expanded on their ideas, filled in gaps, introduced vocabulary, and ultimately aimed toward a predefined set developed in advanced by the instructor. Following this, students were asked: "what is the 'design space' of living systems?" This question, with the instructors help, is designed to illustrate the physical limits imposed upon terrestrial organisms. The 
objectives of these exercises were: 1) elicit and record students' initial concepts, 2) to illustrate the functional aspects of living systems, and introduce the principle that the design space of life is constrained by a rational set of physical parameters, and 3)allow the instructor to establish the framework for the discussions to follow in the course.

Module 2: Introduction to The Human Genome Project and genetic mapping. For this exercise, the students were shown the recently published map of the human genome ${ }^{1}$ and were challenged to decode the meanings of its annotations, both by inspection and by asking the instructor questions. A homework assignment followed this exercise that required the students to study the structure of the DNA double helix and answer a set of questions related to important concepts of DNA structure and function. These exercises introduced the concepts of genomic organization, genes, and DNA structure in the context of understanding the biomedical implications of this major technological accomplishment. An additional homework assignment asked students to contemplate some of the complex ethical issues raised by genetic mapping and sequencing. Their opinions about the ethical implications of genetic testing were discussed during the next class session and stimulated a lively discussion.

Module 3: DNA Forensics - the Anna Anderson story. This module, designed to introduce the central dogma, and principles of heredity and cell architecture, is based on the story of the untimely demise of Czar Nicholas Romanov II and his family ${ }^{2-4}$. Students were told the story of the discovery in 1990 of human remains near Yekaterinburg, Russia, believed, but at the time not yet proven to be those of the Romanovs. The students were also informed of Anna Anderson's claim that she was their living daughter, who's body was missing from the grave site. Students were introduced to the scientific evidence collected from the bodies and from Anna Anderson, and were required to either confirm or refute, based on this evidence, whether 1) the bodies belonged to the Romanovs and 2) whether Anna Anderson was the missing Czarina. Despite the fact that the answer is already known, the students were required to master the concepts of inheritance, DNA variation, genetic polymorphisms, DNA sequencing, and the polymerase chain reaction in order to support and explain their own conclusions. To provide hands-on experience with these techniques, this module also included a lab exercise wherein the students collected samples of their own DNA and performed a PCR reaction using primers commonly used in forensic analysis, giving them direct laboratory experience with PCR, gel electrophoresis, and standard gel data analysis.

Module 4: Osteogenesis Imperfecta - a point mutation that causes systemic disease. This module was designed to teach biology concepts by using disease as a basis for understanding normal tissue function. Students were introduced to real-world data that showed how the strength of bone is dramatically compromised when a point mutation in the collagen gene leads to defective proteins that are unable to assemble into normal triple helices ${ }^{6}$. This module is especially well suited for introducing biological concepts to engineering students, since bone is a composite material which gains its strength not only from the material properties of its protein and mineral components, but also from the structure of the mineralized portion of trabecular bone itself. From within this setting, the engineering students are able to see the direct link between the properties of a purely biological system and abstract engineering principles, and can see what happens to the bones of individuals who carry the disease (students are shown actual Xray data of neonates with OI whose every bone is broken.) This module extends the concepts 
introduced in the previous two modules by drawing on the students' understanding of the central dogma, protein expression, heredity and DNA variation, and the basics of eukaryotic cell architecture. This module also powerfully illustrates the integration of biological systems at various levels.

These examples illustrate how the course planners designed specific course content that establishes relevant, interesting problems from within which to guide and structure their learning of fundamental biological vocabulary, concepts, and systems. They further illustrate how each module builds layers of complexity onto the concepts introduced earlier in the course.

\section{Module Implementation}

A substantial body of research has shown how effective problem-based instructional methods can be in a variety of domains ${ }^{6,7}$. However, designing an introductory course that relies strictly on problem-based exercises can be problematic, particularly courses such as this one that introduce students with minimal biology background to cell and molecular biology, a domain largely outside of common experience. Therefore, we implemented a series of steps designed to introduce just enough knowledge to enable the students to work on open-ended problem-based exercises within each module. The steps are: 1) introduce and explain new material in a standard lecture format, 2) assign homework that requires students to both answer retrospective questions about material introduced in the previous lecture, as well as do their own prospective investigation into the subjects that will be covered in the next period, 3) introduce and explain the module framework (describe the problem), 4) examine the conceptual aspects of the framework in detail, posing increasingly complex questions to build and reinforce knowledge and understanding, and 5) perform related laboratory exercises to enhance learning whenever resources permit.

We believe that this tiered instructional approach addresses specific difficulties sometimes associated with a purely problem-based methods. For instance, the initial lecture and homework assignments effectively introduce basic concepts and vocabulary by means of an instructional format the students are familiar and comfortable with; material that can be understood students with diverse backgrounds. Not only does this lay the groundwork for understanding and analyzing the framework which will be used for the remainder of the module, it serves as an introduction to the resources the students will need throughout the course ${ }^{8}$. By using this instructional approach, we enable students to establish a foundation of confidence in their understanding of new ideas from which to move into more exploratory settings, such as in-class, student- and team-centered, problem-based exercises. Next, from within the context of these exercises, the students are challenged to apply the concepts, tools, and skills to solve problems under expert guidance. Finally, students use their new knowledge and skills to complete a laboratory exercise, which provides an opportunity to ground the principles in a physical, rather than conceptual, setting, and gives them real, hands-on experience to further support their understanding.

\section{Course Evaluation}


The focus of our evaluation was to provide students with feedback and to inform continual curricular development and revision. Specific curriculum revision goals include: 1) documenting, assessing, and understanding changes in students' understanding of biology concepts, 2) assessing students' problem-solving skills and ability to apply knowledge to new situations, 3) monitoring student motivation and interest in pursuing further study, 4) assessing instructional effectiveness, and 5) identifying concepts students are having difficulty understanding. We utilized a combination of assessment and elicitation methods to "triangulate" on changes in students' knowledge, skills, and attitudes as a result of their experience in this course. Triangulation is a particularly effective means for measuring student learning ${ }^{9,10}$.

Table II. Assessment methods used for Spring 2001 course evaluation.

\begin{tabular}{|l|l|l|}
\hline \multicolumn{1}{|c|}{ Assessment Instrument } & \multicolumn{1}{|c|}{ Administered } & \multicolumn{1}{c|}{ Assessment Target } \\
\hline $\begin{array}{l}\text { Open-ended and closed- } \\
\text { ended survey questions }\end{array}$ & Beginning and end of course & $\begin{array}{l}\text { Attitudes about biology; } \\
\text { Self-evaluation and feedback about course content; } \\
\text { Description and explanation of biology concepts; } \\
\text { Student's background }\end{array}$ \\
\hline Word association task & Beginning and end of course & $\begin{array}{l}\text { Recall of biological knowledge (breadth and } \\
\text { sophistication) }\end{array}$ \\
\hline $\begin{array}{l}\text { In-class exercises, exams, } \\
\text { homework }\end{array}$ & Throughout course & Conceptual understanding \\
\hline In-class discussions & Throughout course & Conceptual understanding \\
\hline Class observations & Throughout course & $\begin{array}{l}\text { Instructional strategies and activities; } \\
\text { Course organization }\end{array}$ \\
\hline
\end{tabular}

Table II lists the assessment strategies used, when they were administered, and the focus of the assessment. Every attempt was made to integrate assessment activities into work assigned as a natural part of the course (e.g., homework, exams) to minimize "assessment overhead" on the students' course load. Examples of survey, homework, and exam questions are provided in Table III. For the word association tasks, students were asked to list terms they associate with a specific anchoring concept ${ }^{11}$. Anchoring concepts included "what is biology", "what are the essential functions of life", and "what is heredity".

Table III. Example questions from the surveys, homework, and exams.

\begin{tabular}{|l|c|}
\hline Instrument & Example Questions \\
\hline Survey & Self-assessed understanding of biology (rating from poor (0) to excellent (5)): \\
& I can utilize my understanding of biology to describe a biological system \\
I can explain the relevant biology concepts to another engineer \\
Attitudes about biology (rating from strongly disagree (1) to strongly agree (4)): \\
Biology as memorizing vocabulary or known facts \\
Biologists utilize equations/mathematics skills to describe/solve problems \\
Properties of biological systems can be derived intuitively \\
What I can learn about biology will not relate to what I know about engineering \\
Motivation to enroll in more biology courses (rating from none (0) to very high (5)): \\
As a result of class, interest in pursuing future biology experiences \\
As a result of class, likelihood to pursue future biology experiences \\
Students' assessment of course effectiveness (rating from none (0) to very high (5)): \\
The Human Genome Module contributed to my understanding of DNA \\
The Osteogenesis Imperfecta Module contributed to my understanding of heredity \\
Descriptions and explanations of biology concepts: \\
What were the central concepts discussed in this course?
\end{tabular}

Proceedings of the 2002 American Society for Engineering Education Annual Conference \& Exposition Copyright (C) 2002, American Society for Engineering Education 


\begin{tabular}{|l|l|}
\hline \multirow{7}{*}{ Homework } & $\begin{array}{l}\text { Describe an example of an engineering problem in which understanding of biology might be } \\
\text { useful. What kinds of biology knowledge would you need to solve this problem? } \\
\text { Explain the central dogma as if you were talking to another engineer. How confident are you that } \\
\text { this is a good explanation? } \\
\text { Identify mechanisms of variability in DNA. }\end{array}$ \\
$\begin{array}{l}\text { A stated aim of the OI Module is to 'teach biology from a disease perspective' -- Use your own words } \\
\text { to explain what teaching biology from a disease perspective means. } \\
\text { How does an engineering system compare with a biological system? Which system is more amenable } \\
\text { to quantitative analysis? Please explain. } \\
\text { Consider two forms of atherosclerosis. One form affects children. The other affects adults. Which } \\
\text { form is more likely to be due to genetic mutation? } \\
\text { What is the central dogma? } \\
\text { In a recent NY Times article, Stephen Jay Gould suggested that the central dogma was an overly } \\
\text { simplistic concept that must be modified, especially now that the human genome has been shown } \\
\text { to contain only 35,000 genes. Use your own words to explain Gould's thesis. Do you } \\
\text { agree/disagree with his point of view? }\end{array}$ \\
$\begin{array}{l}\text { Given a forensics scenario about Alexi Romanov: Your task today is to generate a plan for how to } \\
\text { determine whether these remains are those of Alexi Romanov. Students were asked to respond to } \\
\text { the following questions: (1) What can you test? (2) What samples would you hope to obtain and } \\
\text { from whom? (3) What kinds of target genes would you use? (4) What kinds of results would be } \\
\text { definitive or inconclusive, and (5) Analyze your plan for feasibility and reliability. }\end{array}$ \\
\hline Exams
\end{tabular}

\section{Using Course Evaluation to Inform Course Improvement}

Five students enrolled in the Spring 2001 pilot, four senior Chemical Engineering students and one junior Civil and Environmental Engineering student. Results from the pre-test survey indicate that there were noticeable differences in students' backgrounds. For example, only one student had completed a college level biology course whereas four of the students had completed only a high school level biology course. Also, only three of the students had either completed or were currently enrolled in a course that emphasized engineering design. Finally, the Civil Engineering student had completed coursework in dynamics, materials science, and mechanics of materials; the Chemical Engineering students were more likely to have completed coursework in thermodynamics and transport systems. This difference is not surprising given the core requirements for each department, although it highlights the different kinds of engineering knowledge students will bring to an interdisciplinary engineering course such as "Biological Frameworks for Engineers".

Overall, our post-instructional evaluation indicated notable changes in students' 1) beliefs about the nature of biology, 2) recall of biology concepts, and 3) understanding of biology concepts. We also identified difficulties students had describing and explaining biology concepts as well as their ability to apply their understanding of biology concepts to solve specific problems.

Students stated that they enjoyed the course and were interested in pursuing future experiences to learn biology although many said that this was not likely to occur. Students also identified substantial concerns regarding course organization, a lack of an obvious engineering component, and too great of an emphasis on functions associated with DNA over other biology concepts. Therefore, aspects of the modules and the instructional methods were at least partially effective for instructing biology concepts and enhancing students' confidence about their understanding of biology. Given that this was an initial, largely experimental pilot study, it is not surprising that we encountered difficulties. Over the intervening summer quarter, we modified the course 
curriculum and instructional methods to build upon our successes and to implement changes suggested from the assessment data. These changes were implemented in the fall of 2001.

The following examples highlight some of our assessment findings and how they were used to inform curricular development and revision. These examples were derived from a synthesis across findings from the assessment activities identified in Table II.

Students' Understanding of Biology Concepts. Students' recall of biology concepts and selfassessed understanding of biology increased. Comparing across pre- and post-word association responses, there were increases in the total number of concepts (average $=13$ ) and the number of concepts associated with each course module. For example, the average number of concepts from the Anna Anderson module increased by 16 and the number of concepts from the Osteogenesis Imperfecta module increased by 8 . The number of concepts that were course themes essentially doubled, in particular those associated with the central dogma, structurefunction relationships, ethics, and heredity. In addition, the sophistication of terms increased. At the beginning of the course, students identified biology concepts that were largely based on concepts learned from high school biology courses. By the end of the course, students identified terms that were more sophisticated and more representative of the functions of biological systems. Common terms on the pre-test include: DNA, RNA, genes, families, organs, dissecting, and evolution. In comparison, themes more likely to be on the post-test include enzymes, heredity, mutation, systems, mutation polymorphism, central dogma, disease, transcription, translation, and structure-function relationships. For an engineering problem at the biology interface, students perceived an increase by at least one rank on the survey scale in their abilities to 1) identify and understand the relevant biology concepts and 2) utilize an understanding to describe and solve the problem. Students also rated their abilities to explain biology concepts and to describe the relationship between concepts in a biological system as "very good". Finally, students were able to sufficiently describe, explain and apply principles associated with the central dogma and heredity, and to some extent principles of disease and mutation.

Students had difficulty explaining and applying concepts associated with DNA variation and its consequences. A persistent misconception was a belief that small changes in DNA sequence almost always lead to dramatic changes in phenotype. We also found that some of the central course themes were not evident in the word association tasks or in the final course evaluation survey which asked students to list central course concepts. Concepts essentially missing from the word association include: information processing, bioenergetics, biosynthesis, homeostasis and growth, signaling and communication, systems integration, and thermodynamics. Concepts missing from the course evaluation include ecology, structure-function relationships, and bioenergetics.

Data gained from the class observations and discussions, as well as the surveys, provide some insight into the nature of these problems. For instance, some of the course activities which were designed to elicit active thinking and application of concepts appeared to be too open-ended or obscure for the students. An example of this is "The Introduction to the Human Genome Project" exercise. Our observations suggest that this exercise was unsuccessful at fostering student interest and interaction as evidenced by the limited scope and range of the questions asked by students during this module. One possible reason may be that students lacked sufficient 
knowledge about genome organization and DNA structure to generate ideas, questions, or develop an understanding. . Assessments of students' homework and exams also indicate that most of the students had difficulty understanding some of the important content objectives. These findings suggest areas for future improvement. In particular, revisiting specific content or utilizing alternative teaching strategies to ensure student learning, and perhaps even abandoning content objectives that may not be appropriate for this audience and course. Observations also suggest that cell signaling, bioenergetics, and ecology concepts were not adequately covered.

A number of changes to address these problems were implemented in the Fall 2001 course offering. A specific change to the DNA Forensics module was adding a computer modeling exercise on DNA binding proteins. This exercise gave the students an opportunity to visualize the three-dimensional structure of DNA and a DNA binding protein, and investigate how the two molecules might interact. This exercise reinforced students' knowledge of the components and structure of DNA and proteins and the relationship between their structure and function, as well as introducing protein structure databases and the capabilities of computerized modeling software. It and was followed up with homework that encouraged them to explore the specific details of DNA and protein structure and the important interactions between them.

Another specific change made to the DNA forensics-Anna Anderson module was to include a detailed lecture on the mechanisms of DNA variation which is a cornerstone topic to all the course modules and a specific weakness identified in the pilot course. To address missing content associated with ecology concepts, a third module based on bioremediation was developed and implemented. Content for the Bioremediation / Environmental Engineering module was centered about a laboratory exercise involving enriching and isolating a phenoldegrading organism for the environment. As such, related biology concepts include cellular energetics, metabolic diversity, and ecology.

Articulating a Biological Perspective. As a group, the students moved towards a broader biological perspective. By the end of the course, the range of variation across students' beliefs about biology decreased and students were more likely to describe biological systems as complex systems that have many unknowns and are governed by fundamental physical principles. Students became stronger in their beliefs about there being more than one interpretation for a given biological phenomenon, and two students changed their beliefs about "biology as intuitive" from agree to disagree (the remaining students generally disagreed with this statement). On the post-survey, students could articulate some important differences, as well as some similarities, between engineering and biological perspectives. For example, students were more likely to describe biology problems as more complex and explained that less is generally known about biological systems than engineering systems.

However, students" misconceptions about what biologists "do" and how their research methods differ from those used by engineers persisted. Here, "misconceptions" represent an instance when a student does not have an expert-like understanding of a concept. Students tended to describe the relevance of biological principles for engineering problems as minimizing environmental impact of human activity on biological systems. More importantly, half of the students agreed and half disagreed with a statement that "biologists utilize mathematical equations to describe biology concepts". This suggests that students were confused about the 
nature of biology as a science that can be described using fundamental principles. A persistent misconception evident in both the surveys and homework was that engineers apply quantitative techniques whereas biological systems are not readily amenable to quantification. One student even described methods for studying biological systems as "passive and memorization". Class observations reveal that mathematical models and techniques for studying biological systems were not discussed. Given that an understanding of mathematical principles is essential for engineering students, implementing more opportunities for students to explore mathematical models of biological systems is indicated for our course.

To address this shortcoming, a substantial modification was incorporated into the Fall 2001 course. Each module was revised to include an exercise that either models a biological system response or uses computer modeling of bio-macromolecules to illustrate a biological principle. For the DNA forensics- Anna Anderson module a computer simulation activity for modeling a transcriptional feedback mechanism was designed and implemented. This exercise integrated the students' familiarity with mathematical modeling, differential equations, MATLAB programming, and control loops to examine potential biological responses to a variety of different input parameters. The Osteogenesis Imperfecta module was revised to have a stronger emphasis on the process of life science discovery and the differences between engineered and living systems. A classroom demonstration of data transformation and interpretation was also added. In this activity, a graduate student led a discussion illustrating how to transform and interpret digital image data she had collected to confirm a model of bone growth. Using computer software to do real-time image analysis and projecting the resulting plots on a screen, the graduate student led the group through the process of normalizing and analyzing actual experimental data. During this demonstration, the class was able to receive immediate feedback on how well their suggestions, based on their developing understanding of the problem, would enable them to perform a meaningful analysis on the raw data. Eventually, the students were asked to support or refute a specific biological hypothesis using the data they developed in class.

Course Organization / Integration of Concepts. Overall, students responded that they enjoyed the problem-based learning approach, hands-on activities, classroom discussions, and course handouts. In particular, students commented that they learned a great deal from the laboratory exercises and that a particular strength of the course was the opportunity to engage in small group discussions and interact with the instructors in an intimate setting. Class observations also suggest that the ethics discussions stimulated a high level of interest and active discussion.

Students also expressed dissatisfaction with the course organization, some of the content, and the integration of content across the modules. More specifically, students commented that too much material was covered for sufficient understanding; problems regarding the vocabulary for each module were difficult to overcome; and the connections across modules and between biology and engineering concepts were not well articulated. Our class observations confirm that these were areas of potential improvement to the course.

Changes implemented in the Fall 2001 course offering consist of building on the existing strengths of the course and addressing the identified challenges. The success of the PCR lab as a hands-on learning experience prompted a decision to include at least one lab exercise in each module. To address difficulties associated with understanding the vocabulary, a vocabulary 
activity was assigned prior to each module in which students define a list of vocabulary terms. Also, to ease the transition to biology and emphasize the relationships between engineering and biology concepts, biological processes were introduced or explained whenever possible by drawing analogies to engineering systems. As an example, a specific change to The Functions of Life Module was to implement a small group activity where students discuss comparisons between biological and engineered systems; for example, a robotic weather drone aircraft and a seagull. Students were asked to list their answers, and this list was condensed into a summary of the essential functions of life. As such, the instructional objective was to engage students in thinking about both the differences and the similarities between human-engineered and biological systems.

To address concerns about course cohesiveness and organization, we created a framework to inform the design of lectures and classroom activities. This content map is illustrated in Figure 1. As shown here, the map highlights important course content and how they related to individual modules as well as across modules.

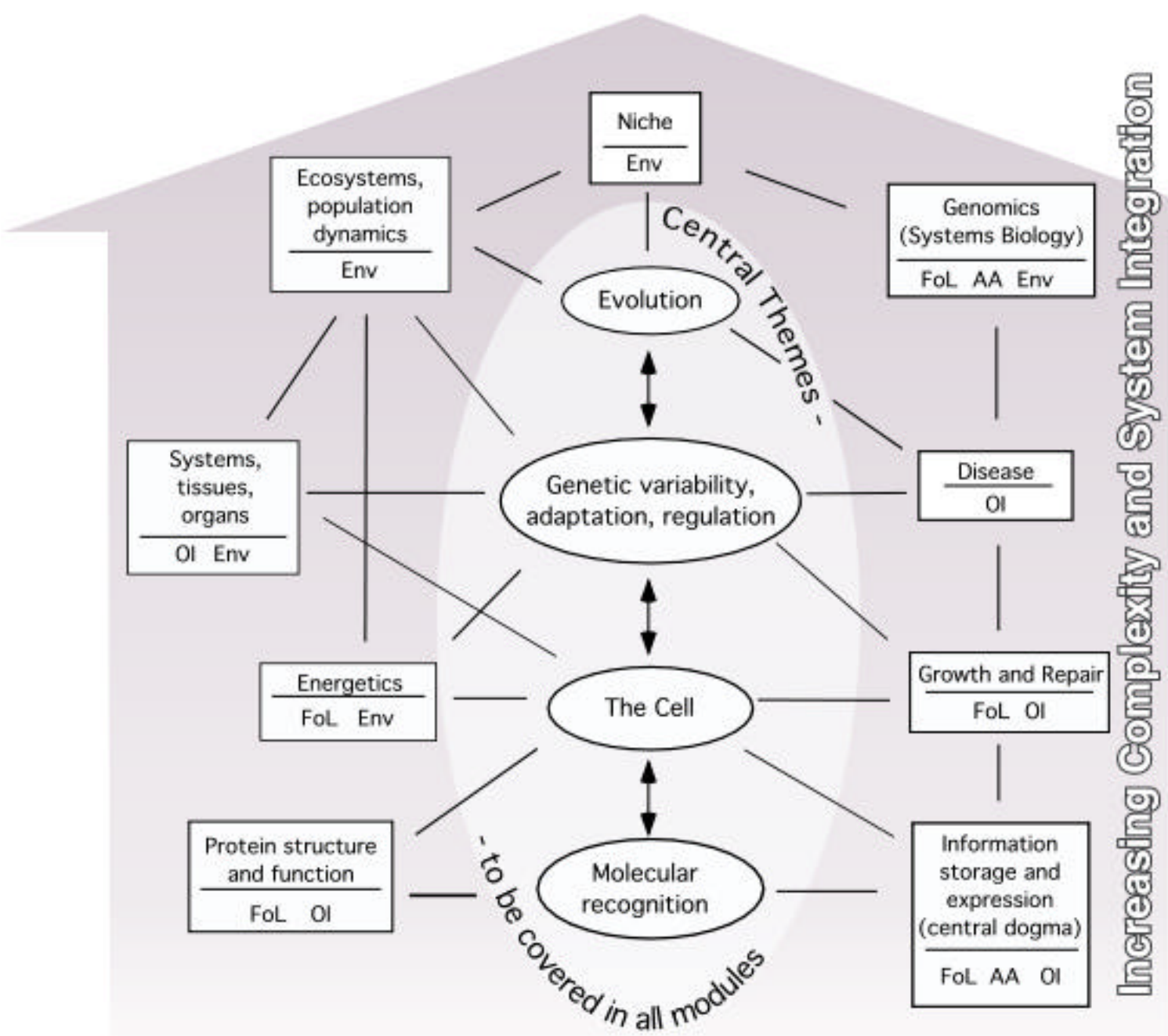

Figure 1: Biological Frameworks for Engineers course content map. Essential concepts (in ovals) are discussed and reinforced in each module. Additional content (boxes) are covered in individual modules. Content is stratified on map and levels of "increasing complexity"; systems higher on the map are built upon systems underneath. Fol- 
functions of life; AA - Anna Anderson (DNA forensics); OI - Osteogenesis Imperfecta; Env - Environmental Engineering

And finally, to help students integrate all the key concepts introduced in the course and challenge the students to apply the biological principles they've learned in an engineering setting, the autumn quarter offering included a term long design project focused on identifying the problems and risks associated with a manned mission to Mars, then proposing and evaluating solutions to the issues they've identified using concepts discussed during the course.

Effectiveness of the Assessment Instruments. Our assessment strategy for documenting and eliciting changes in students' beliefs and understanding of biology as a means of informing curricular revision was effective. Weaknesses were identified, however. Assessment instruments administered at the beginning and end of the quarter were modified as follows: 1) questions that provided little insight were removed, 2) questions that were poorly worded were revised, 3) questions to gain greater insight into persistent misconceptions were expanded, and 4) questions about important concepts for each individual module were added. We also realized that our existing instruments did not adequately assess students' ability to differentiate and integrate their understanding of biology. As a result, we extended the word association task to include a concept map activity. Students were asked to list terms they associated with the "essential functions of life" and use this list to create a node and arc representation of how terms related ${ }^{11}$. Finally, we plan to analyze laboratory results and reports to assess students' understanding of how mathematical models can be used to describe biology concepts.

\section{Summary}

This paper describes a course, Biological Frameworks for Engineers, designed to teach biology to engineering undergraduate students. Our course evaluation indicates that this course has been effective in meeting most of its stated learning objectives. More importantly, we have utilized our assessment findings to improve the course curriculum and instructional methods.

Preliminary findings from our second iteration of the course indicate that 1) students' understanding and use of concepts related to the central themes of the course markedly improved and 2) students' ability to identify common course themes was greatly enhanced. Preliminary student feedback suggests that the use of analogies drawn between engineering and biological systems was helpful for introducing students to new concepts. Also, data from our course observations reveal dramatic successes associated with lecture and demonstrations drawn from the instructors own research projects.

Overall, successful features of our course design and evaluation strategy include:

- Teaching biology from an engineering perspective

- Basing curriculum within a real-world "framework" to enhance a feeling of relevance and structure

- Utilizing a combination of pedagogical strategies ranging from lectures, labs, and problem-based learning exercises to facilitate the introduction of biology concepts to engineering undergraduates

- Integrating content and assessment

- Implementing an iterative development process 
Ultimately, this iterative development process will aid us in our efforts to produce modules that can be readily exported to other universities for integration into their programs.

\section{Acknowledgements}

The authors wish to thank Heather Rothfuss and especially Kelly Fitzgerald for classroom support, Dr. Paolo Vicini for his contributions to the mathematical modeling exercises, Dr. Martin Kushmerick for his generous advice and support with the "Mission to Mars" term project, Jack Herndon for laboratory support, Allen Olsen for his help with the pilot course development, Dr. Angela Linse for her assistance with establishing guidelines for creating course learning objectives. Funding for this work was provided by NSF \#0080364 (Integrating Biology into the Engineering Curriculum at the University of Washington).

\section{References}

[1] McPherson, J. D. et al.(2001), “A physical map of the human genome”, Nature 409(6822):934-41

[2] Editorial (1994). “Anastasia and the tools of justice.” Nature Genetics 8(3): 205-206.

[3] Editorial (1996). "Romanovs find closure in DNA.” Nature Genetics 12(4): 339-340.

[4] Stoneking, M., T. Melton, et al. (1995). "Establishing the Identity of Anna Anderson Manahan." Nature Genetics 9: 9-10.

[5] Byers, P. H. (2000). "Osteogenesis imperfecta: perspectives and opportunities." Current Opinion in Pediatrics 12: 603-609.

[6] Wilkerson, L. and W. H. Gilselaers (1996). Bringing problem-based learning to higher education; theory and practice. San Francisco, Jossey-Bass.

[7] Bransford, J., Brown A., and Cocking R. (eds.) (2002). How People Learn: brain, mind, experience, and school (expanded edition) Washington D.C., National Academy Press

[8] We have selected supplementary resources on the World Wide Web, which allows us to tailor readings to the specific framework rather than tailoring the coursework to a textbook. Many excellent resources are already published on the Internet. See, for example,

www.uic.edu/classes/phar/phar331

www.ks.uiuc.edu/Research/vmd

www.rcsb.org/pdb

http://chemistry.gsu.edu/glactone/PDB/pdb.html

http://hope-dna.com/docs/fbi kids dnapage.htm

www.attorneys.com.mx/english/dnatesting.html 
http://users.netaccess.co.nz/chocfish/histpage_rrdna.html

www.euro.promega.com/geneticidprod/ussymp8proc/ab25.html

http://esg-www.mit.edu:8001/esgmio/7001main.html

http://esg-www.mit.edu:8001/esgmio/lm/lmdir.htm

www.bartleby.com $/ 107 / 18 / \mathrm{html}$

www.sciencemag.org/cgi/content/full/291/5507/1224

www.nap.edu/books/0309073197/html/index.html

www.cellsalive.com

www.cbs.dtu.dk/dave/DNA_CenDog.html

http://campus.northpark.edu/biology/cell/basiccell.html

[9] Atman, C. J., R. S. Adams, et al. (2000). Using Multiple Methods to Evaluate a Freshman Design Course. 30th ASEE/IEEE Frontiers in Education Conference, Kansas City, MO.

[10] McGourty, J. (1999). "Four Strategies to Integrate Assessment into the Engineering Educational Environment." Journal of Engineering Education 88(4): 391-395.

[11] Turns, J., Atman, C.J., \& Adams, R., "Concept maps for engineering education: A cognitively motivated tool supporting varied assessment functions", IEEE Transactions on Education, May 2000. 